\title{
Palmitoylethanolamide and Its Biobehavioral Correlates in Autism Spectrum Disorder: A Systematic Review of Human and Animal Evidence
}

\author{
Marco Colizzi ${ }^{1,2,3, *(D)}$, Riccardo Bortoletto ${ }^{1}$, Rosalia Costa ${ }^{4}$ and Leonardo Zoccante ${ }^{1}$ \\ 1 Child and Adolescent Neuropsychiatry Unit, Maternal-Child Integrated Care Department, \\ Integrated University Hospital of Verona, 37126 Verona, Italy; riccardo.bortoletto91@gmail.com (R.B.); \\ leonardo.zoccante@aovr.veneto.it (L.Z.) \\ 2 Section of Psychiatry, Department of Neurosciences, Biomedicine and Movement Sciences, \\ University of Verona, 37134 Verona, Italy \\ 3 Department of Psychosis Studies, Institute of Psychiatry, Psychology and Neuroscience, \\ King's College London, London SE5 8AF, UK \\ 4 Community Mental Health Team, Department of Mental Health, ASST Mantova, 46100 Mantua, Italy; \\ rosalia.costa80@gmail.com \\ * Correspondence: marco.colizzi@univr.it; Tel.: +39-045-812-6832
}

check for updates

Citation: Colizzi, M.; Bortoletto, R.; Costa, R.; Zoccante, L.

Palmitoylethanolamide and Its Biobehavioral Correlates in Autism Spectrum Disorder: A Systematic Review of Human and Animal Evidence. Nutrients 2021, 13, 1346. https://doi.org/10.3390/nu13041346

Academic Editors: Ludovica Pasca and Pierangelo Veggiotti

Received: 17 March 2021

Accepted: 16 April 2021

Published: 18 April 2021

Publisher's Note: MDPI stays neutral with regard to jurisdictional claims in published maps and institutional affiliations.

Copyright: (c) 2021 by the authors. Licensee MDPI, Basel, Switzerland. This article is an open access article distributed under the terms and conditions of the Creative Commons Attribution (CC BY) license (https:/ / creativecommons.org/licenses/by/ $4.0 /)$.

\begin{abstract}
Autism spectrum disorder (ASD) pathophysiology is not completely understood; however, altered inflammatory response and glutamate signaling have been reported, leading to the investigation of molecules targeting the immune-glutamatergic system in ASD treatment. Palmitoylethanolamide (PEA) is a naturally occurring saturated $\mathrm{N}$-acylethanolamine that has proven to be effective in controlling inflammation, depression, epilepsy, and pain, possibly through a neuroprotective role against glutamate toxicity. Here, we systematically reviewed all human and animal studies examining PEA and its biobehavioral correlates in ASD. Studies indicate altered serum/brain levels of PEA and other endocannabinoids (ECBs)/acylethanolamines (AEs) in ASD. Altered PEA signaling response to social exposure and altered expression/activity of enzymes responsible for the synthesis and catalysis of ECBs/AEs, as well as downregulation of the peroxisome proliferator activated receptor- $\alpha$ (PPAR- $\alpha$ ) and cannabinoid receptor target GPR55 mRNA brain expression, have been reported. Stress and exposure to exogenous cannabinoids may modulate ECBs/AEs levels and expression of candidate genes for neuropsychiatric disorders, with implications for ASD. Limited research suggests that PEA supplementation reduces overall autism severity by improving language and social and nonsocial behaviors. Potential neurobiological underpinnings include modulation of immune response, neuroinflammation, neurotrophy, apoptosis, neurogenesis, neuroplasticity, neurodegeneration, mitochondrial function, and microbiota activity, possibly through peroxisome proliferator-activated receptor- $\alpha$ (PPAR- $\alpha)$ activation.
\end{abstract}

Keywords: neurodevelopment; pervasive developmental disorder; cannabinoids; acylethanolamines; immune response; glutamate; inflammation; peroxisome proliferator-activated receptor- $\alpha$; child and adolescent neuropsychiatry

\section{Introduction}

Autism spectrum disorder (ASD) is a complex and multifactorial neurodevelopmental condition affecting those who suffer from it at different levels. Difficulties in social communication and associated tendency to have restrictive interests and repetitive and stereotyped activities are considered the core aspects of the disorder [1], allowing clinicians to make a diagnosis of ASD based on behavioral assessment [2]. However, converging research evidence supports the presence of altered neurobiological parameters such as functional and structural brain abnormalities $[3,4]$, by which gene variants or epigenetic marks lead to the neurocognitive and biobehavioral symptoms consistently reported in ASD [5,6]. It is 
noteworthy that, even though the pathophysiology of ASD is not completely understood, studies conducted over the last two decades have helped clarify some of the mechanisms of disease progression. In particular, altered inflammatory response [7] and disrupted glutamate signaling [8-10] have been reported, leading the way to the investigation of the effect of molecules targeting the immune-glutamatergic system in improving clinical severity in ASD [11].

Growing evidence indicates that one of the major physiological functions of the cannabinoid signaling system is the modulation of neuroinflammation [12] and glutamate neurotransmission [13]. Both exogenous cannabinoids (e.g., plant-derived cannabinoids $\Delta 9$ tetrahydrocannabinol, $\triangle 9-\mathrm{THC}$, and cannabidiol, CBD) and their endogenous counterparts (e.g., endocannabinoids anandamide and 2-arachidonoylglycerol, 2-AG) interact with the endocannabinoid system, with implications for health and disease [14,15]. Despite promising evidence on the efficacy of $\triangle 9$-THC- and CBD-based treatments in the management of ASD-related behavioral problems, it is still limited and requires further investigation [16]. Anandamide (arachidonoylethanolamide) and 2-AG are both derivatives of arachidonic acid, a polyunsaturated fatty acid well known as the precursor of bioactive prostaglandins and other eicosanoids, with anandamide specifically being its ethanolamine [17].

Other ethanolamines of various long-chain fatty acids also naturally occur, and along with anandamide they are collectively referred to as $\mathrm{N}$-acylethanolamines. However, they are more abundant than anandamide in the body and do not bind to cannabinoid receptors, while exerting most of their biological effects by activating the peroxisome proliferatoractivated receptor- $\alpha$ (PPAR- $\alpha$ ), a nuclear receptor, and PPAR- $\alpha$-independent pathways involving other receptors such as Transient Receptor Potential Vanilloid 1 (TRPV1) and GPR55 [17,18]. Research evidence suggests that such specific mechanisms of action would account for their anti-inflammatory, analgesic, anticonvulsant, and neuroprotective properties [17,18]. Palmitoylethanolamide (PEA, N-hexadecanoylethanolamide), in particular, is a saturated $\mathrm{N}$-acylethanolamine which has been suggested to be effective in the control of inflammatory responses [19,20], depressive symptoms [21], epilepsy [22], and pain [23], possibly through a neuroprotective role against glutamate toxicity [17].

\section{Objectives}

This effect of PEA on (neuro)inflammation and glutamate signaling could represent a promising neurobiological mechanism underlying the clinical utility of such $\mathrm{N}$ acylethanolamine in ASD. This review aims to bring together and discuss all available data generated by clinical and preclinical studies investigating the role of PEA in ASD by conducting a systematic literature search for all such data. We reviewed all interventional and observational studies, employing either retrospective or prospective methodological approaches with any PEA biobehavioral correlates investigated in ASD.

\section{Experimental Procedures}

\subsection{Inclusion and Exclusion Criteria}

To summarize previous research evidence on the topic, inclusion criteria for studies were: (1) human or animal studies, (2) studies investigating both the acute and long-term effects of palmitoylethanolamide (PEA) administration in autism spectrum disorder (ASD) and related conditions, (3) studies investigating PEA signaling-related molecular markers in ASD and related conditions, including (a) blood serum levels, (b) brain tissue levels, (c) enzyme activity, and (d) receptors. Exclusion criteria were (1) studies where PEA was not the intervention of interest (i.e., studies evaluating only exogenous cannabinoid agonists or antagonists), (2) studies where PEA biobehavioral correlates were not investigated with reference to ASD, and (3) studies in which the PEA biobehavioral correlates were not directly reported on. 


\subsection{Search Strategy and Data Extraction}

A literature search was conducted using electronic databases (MEDLINE, Web of Science and Scopus) for any published original study written in English, using a combination of search terms describing autism ('autism spectrum disorder,' 'Asperger syndrome,' 'pervasive developmental disorder,') and palmitoylethanolamide ('palmitoylethanolamide,' 'palmitylethanolamide', 'N-(2-hydroxyethyl)hexadecanamide', 'N-(2-hydroxyethyl)palmitate', 'N-palmitoylethanolamine') on 3 March 2021. Reference lists of eligible studies were also screened to identify additional eligible research. Publication data screening and extraction were performed following a 2-step selection process (conventional double-screening) conducted by 2 reviewers independently of each other (MC and $\mathrm{RB}$ ).

\subsection{Risk of Bias}

The methodological heterogeneity of the studies (Table 1) included in this review necessitate a suitably inclusive and flexible approach to assess risk of bias and study quality. Thus, we used an adapted set of criteria suggested by the Agency for Healthcare Research and Quality (AHRQ) guidance [24], amended as appropriate for interventional and observational studies in humans (Table 2). Risk of systematic bias across human studies was additionally assessed by screening all papers for potential confounding variables such as comorbid psychiatric conditions and substance use (Table 2). Further, to assess any factor that may account for similarities and differences between animal studies, information was extracted about study characteristics, including animal model (e.g., mouse or rat), autism model (e.g., valproic acid, perinatal asphyxia, strain of idiopathic autism), developmental stage (i.e., prenatal or postnatal), sex, and PEA dosage (Table 3).

\section{Results}

\subsection{Study Selection}

In summary, 53 records were retrieved. Abstracts of all records were screened against the inclusion and exclusion criteria. After also excluding duplications (Figure 1), a final list of 10 studies ( 3 human, 6 animal, 1 using both humans and animals) was used for systematic analysis in this review (Table 1). In total, the eligible studies assessed different aspects of the palmitoylethanolamide (PEA) signaling pathway (Table 1). These include (1) in vivo PEA treatment exposure in human(s) with autism spectrum disorder by employing a monotherapy design (ASD; two studies; Table 2); (2) in vivo PEA treatment exposure in human(s) with ASD by employing an add-on design (one study; Table 2); (3) PEA quantitative blood assessment in human(s) with ASD (one study; Table 2); (4) in vivo PEA postnatal exposure in animal models of autism (two studies; Table 3); (5) in vivo PEA postnatal exposure in animal models of perinatal brain disorders (two studies; Table 3); (6) PEA quantitative brain assessment in animal models of autism (one study; Table 3); (7) PEA quantitative brain assessment in animal models of stress following exposure to exogenous cannabinoid agonists/antagonists (one study; Table 3); (8) PEA-related enzymes and receptors quantitative brain assessment in animal models of perinatal brain disorders (one study; Table 3). Additional data on methodological quality of studies conducted in humans and animals are reported in Tables 2 and 3. A brief synthesis of the main results is presented below. 
Table 1. Summary of studies investigating palmitoylethanolamide and its biobehavioral correlates in autism spectrum disorder.

\begin{tabular}{|c|c|c|c|c|c|c|}
\hline $\begin{array}{l}\text { Study } \\
\text { (Country) }\end{array}$ & Aim of Study & PEA Type of Study & Population & $\mathbf{N}$ & $\begin{array}{l}\text { Outcome Measure } \\
\text { (Test Name or Description) }\end{array}$ & Results \\
\hline $\begin{array}{l}\text { Antonucci et al. } \\
\text { (2015) (Italy) }\end{array}$ & $\begin{array}{l}\text { To assess the effect } \\
\text { of PEA on } \\
\text { language, behavior, } \\
\text { and immune } \\
\text { chemistry }\end{array}$ & $\begin{array}{l}\text { In vivo treatment } \\
\text { exposure in humans }\end{array}$ & 2 ASD adolescents & 2 & $\begin{array}{l}\text { 1. Expressive language (MLU); } 2 \text {. } \\
\text { overall autism severity (CARS-2; } \\
\text { ATEC); } 3 \text {. immune response } \\
\text { (blood tests) }\end{array}$ & $\begin{array}{l}\text { 1. MLU: } \uparrow \text { from } 3.0 \text { to } 5.4 ; 2 \text {. CARS- } 2 \text { test: } \downarrow \text { from } 43.5 \\
\text { to } 32 \text {, ATEC: } \downarrow \text { from } 25 \text { to } 12 ; 3 \text {. vitamin D-OH25 level: } \\
\uparrow, \text { CD57+ NK: } \uparrow, \text { total lymphocytes: } \downarrow \text {, total serum IgE: } \\
\downarrow \text { by } \sim 50 \% \text { (only in } 1 \text { case, in the other case NS), atopic } \\
\text { illnesses: } \downarrow \text {, total WC: unchanged }\end{array}$ \\
\hline $\begin{array}{l}\text { Aran et al. } \\
\text { (2019) (Israel) }\end{array}$ & $\begin{array}{l}\text { To assess PEA and } \\
\text { other ECBs/AEs } \\
\text { blood levels and } \\
\text { their association } \\
\text { with behavior }\end{array}$ & $\begin{array}{l}\text { Quantitative blood } \\
\text { assessment in humans }\end{array}$ & $\begin{array}{l}\text { 1. } 93 \text { ASD chil- } \\
\text { dren/adolescents; } 2 \text {. } \\
93 \mathrm{HCs}\end{array}$ & 186 & $\begin{array}{l}\text { Serum ECBs / AEs levels } \\
\text { (LC-MS/MS) }\end{array}$ & $\begin{array}{l}\text { 1. Serum levels of AEA, OEA and PEA: ASD < HC } \\
\text { (after Bonferroni correction, still significant), 2-AG: } \\
\text { ASD vs. HC, NS; } 2 \text {. } \downarrow \text { age or } \downarrow \text { BMI: } \downarrow \text { serum levels of } \\
\text { AEA (after Bonferroni correction, NS; correlations } \\
\text { with other ECBs / AEs, NS); } 3 \text {. } \uparrow \text { APSI severity score } \\
\text { or } \uparrow \text { use of antipsychotics: } \downarrow \text { serum levels of AEA } \\
\text { (trend effect; correlations with other ECBs/AEs } \\
\text { and/or other symptoms, NS) }\end{array}$ \\
\hline $\begin{array}{l}\text { Bertolino et al. } \\
\text { (2016) (Italy) }\end{array}$ & $\begin{array}{l}\text { To assess the effect } \\
\text { of PEA on behavior }\end{array}$ & $\begin{array}{l}\text { In vivo treatment } \\
\text { exposure in humans }\end{array}$ & 1 ASD child & 1 & $\begin{array}{l}\text { 1. Overall autism severity (ATEC); } \\
\text { 2. Motor stereotypic behaviors } \\
\text { (Quarterly questionnaire) }\end{array}$ & $\begin{array}{l}\text { 1. ATEC: } \downarrow \text { of both total and subgroup scores (Speech, } \\
\text { Sociability, Sensory/Cognitive, } \\
\text { Health/Physical/Behavior; improved of about } 23 \% \text { ); } 2 \text {. } \\
\text { motor stereotypies: } \downarrow \text {; enuresis: } \downarrow \text { from } 91 \% \text { to } 2.4 \% \\
\text { after } 14 \text { months }\end{array}$ \\
\hline $\begin{array}{l}\text { Khalaj et al. } \\
\text { (2018) (Iran) }\end{array}$ & $\begin{array}{l}\text { To assess the effect } \\
\text { of PEA add-on to } \\
\text { risperidone on } \\
\text { language and } \\
\text { behavior }\end{array}$ & $\begin{array}{l}\text { In vivo treatment } \\
\text { exposure in humans }\end{array}$ & $\begin{array}{l}\text { 1. } 31 \text { ASD (Arm A, } \\
\text { risperidone + PEA); } \\
\text { 2. } 31 \text { ASD children } \\
\text { (Arm B, risperidone + } \\
\text { placebo) }\end{array}$ & $\begin{array}{l}62 \\
\text { (out of } \\
70 \text { ran- } \\
\text { dom- } \\
\text { ized) }\end{array}$ & Behavior (ABC-C) & $\begin{array}{l}\text { 1. ABC-C over time: irritability and hyperactivity, } \\
\text { risperidone + PEA < risperidone + placebo (other } \\
\text { domains NS); } 2 \text {. ABC-C at } 10 \text { weeks: irritability, } \\
\text { hyperactivity, and inappropriate speech (trend), } \\
\text { risperidone + PEA < risperidone + placebo (other } \\
\text { domains NS); } 3 \text {. ABC-C at } 5 \text { weeks: hyperactivity, } \\
\text { stereotypic behavior (trend), and inappropriate } \\
\text { speech (trend), risperidone + PEA < risperidone + } \\
\text { placebo (other domains NS); } 4 \text {. ABC-C response rate } \\
\text { at } 10 \text { weeks, hyperactivity, irritability, and } \\
\text { inappropriate speech, risperidone + PEA > } \\
\text { risperidone + placebo (other domains NS); } 5 \text {. ABC-C } \\
\text { response rate at } 5 \text { weeks, NS; Adverse events, NS }\end{array}$ \\
\hline
\end{tabular}


Table 1. Cont.

\begin{tabular}{|c|c|c|c|c|c|c|}
\hline $\begin{array}{l}\text { Study } \\
\text { (Country) }\end{array}$ & Aim of Study & PEA Type of Study & Population & $\mathbf{N}$ & $\begin{array}{l}\text { Outcome Measure } \\
\text { (Test Name or Description) }\end{array}$ & Results \\
\hline $\begin{array}{l}\text { Bertolino et al. } \\
\text { (2016) (Italy) }\end{array}$ & $\begin{array}{l}\text { To assess the effect } \\
\text { of PEA on behavior, } \\
\text { neuroinflamma- } \\
\text { tion, } \\
\text { neuromodulation, } \\
\text { and neurogenesis }\end{array}$ & $\begin{array}{l}\text { In vivo postnatal } \\
\text { exposure in animals }\end{array}$ & $\begin{array}{l}\text { 1. } 60 \text { SHAM+VHI; } 2 . \\
60 \text { SHAM+PEA; } 3.60 \\
\text { VPA+VHI; } 60 \\
\text { VPA+PEA }\end{array}$ & 240 & $\begin{array}{l}\text { 1. Behavior (SIT; EPM); } 2 . \\
\text { Neuroinflammation, } \\
\text { neuromodulation, and } \\
\text { neurogenesis } \\
\text { (Immunohistochemistry } \\
\text { (Chymase, Tryptase, TNF- } \alpha \text {, } \\
\text { IL-1 } \beta \text { ); Western Blot (Bax, Bcl-2, } \\
\text { iNOS, IkB } \alpha, \text { NF-kB, GFAP); } \\
\text { Neurogenesis (BrdU and DCX } \\
\text { Immunohistochemistry and Golgi } \\
\text { impregnation)) }\end{array}$ & $\begin{array}{l}\text { 1. SIT: stay duration in stranger side, VPA }< \\
\text { VPA+PEA; stay duration in central area, VPA > } \\
\text { VPA+PEA; SI, VPA < VPA+PEA; EPM: time spent in } \\
\text { the open arm, VPA < VPA+PEA; } 2 \text {. chymase, } \\
\text { tryptase, IL-1 and TNF- } \alpha \text { expression to mast cells in } \\
\text { hippocampus and cerebellum: VPA > VPA+PEA; } \\
\text { iNOS and GFAP levels in hippocampus and } \\
\text { cerebellum: VPA > VPA+PEA; IKB } \alpha \text { and NF-kB } \\
\text { levels: VPA < VPA+PEA; Bax: VPA > VPA+PEA; } \\
\text { Bcl-2: VPA < VPA+PEA; BrdU+ cells, DCX+ cells } \\
\text { and development of dendritic spines in the dentate } \\
\text { gyrus of hippocampus: VPA < VPA+PEA }\end{array}$ \\
\hline $\begin{array}{l}\text { Kerr et al. } \\
\text { (2013) (Ireland) }\end{array}$ & $\begin{array}{l}\text { To assess PEA and } \\
\text { other ECBs/AEs } \\
\text { brain levels and } \\
\text { their association } \\
\text { with behavior, and } \\
\text { ECBs/AEs-related } \\
\text { gene expression } \\
\text { and enzyme } \\
\text { activity }\end{array}$ & $\begin{array}{l}\text { Quantitative brain } \\
\text { assessment in animals }\end{array}$ & $\begin{array}{l}\text { 1. } 16 \text { saline; } 2.14 \\
\text { VPA }\end{array}$ & 30 & $\begin{array}{l}\text { 1. Brain ECBs / AEs levels } \\
\text { (LC-MS/MS); } 2 \text {. behavior } \\
\text { (sociability test); } 3 \text {. gene } \\
\text { expression (real-time PCR); } 4 \text {. } \\
\text { enzyme activity }\end{array}$ & $\begin{array}{l}\text { 1. AEA, 2-AG, PEA and OEA in frontal cortex, } \\
\text { hippocampus and cerebellum: VPA vs. saline NS; } 2 \text {. } \\
\text { AEA, OEA and PEA in hippocampus after } \\
\text { sociability test: VPA > saline (other ECBs in other } \\
\text { brain regions NS); } 3 \text {. MAGL mRNA in the } \\
\text { hippocampus, DAGL } \alpha \text { mRNA in the cerebellum, } \\
\text { VPA < saline (other brain region NS); PPAR } \alpha \text { and } \\
\text { PPAR } \gamma \text { mRNA in frontal cortex and hippocampus, } \\
\text { VPA < saline; GPR55 mRNA in frontal cortex and } \\
\text { hippocampus, VPA < saline (cerebellum NS); } \\
\text { CB }_{1} / C_{2} \text { receptor mRNA in frontal cortex, } \\
\text { hippocampus and cerebellum, VPA vs. saline, NS; } 4 \text {. } \\
\text { MAGL activity in the hippocampus, VPA > saline } \\
\text { (FAAH NS) }\end{array}$ \\
\hline $\begin{array}{l}\text { Blanco et al. } \\
\text { (2015) (Spain) }\end{array}$ & $\begin{array}{l}\text { To assess } \\
\text { ECBs/AEs-related } \\
\text { enzyme and } \\
\text { receptor activity }\end{array}$ & $\begin{array}{l}\text { Quantitative brain } \\
\text { assessment in animals }\end{array}$ & 1.CTL; 2. C+;3. PA & $\begin{array}{l}15 \\
\text { (moth- } \\
\text { ers) }\end{array}$ & $\begin{array}{l}\text { 1. Brain ECBs / AEs-related } \\
\text { enzyme and receptor activity } \\
\text { (immunohistochemistry, } \\
\text { immunostaining quantification) }\end{array}$ & $\begin{array}{l}\text { 1. NeuN: NS; GFAP-positive cells: PA > CTL and C+ } \\
\text { in CA1, CA3, and DG regions of dorsal } \\
\text { hippocampus; DAGL } \alpha \text { expression: PA and C+ > } \\
\text { CTL in CA1, C+ > CTL in DG (other regions or } \\
\text { groups NS); NAPE-PLD expression: PA < C+ and } \\
\text { CTL in CA1 and CA3, PA < C+ in DG (other regions } \\
\text { or groups NS); CB1 expression, NS; PPAR } \alpha \text {-positive } \\
\text { cells: PA < C+ and CTL in CA1 and CA3 (DG NS); } \\
\text { FAAH expression: C+ > CTL and PA in CA3 (other } \\
\text { regions or groups NS) }\end{array}$ \\
\hline
\end{tabular}


Table 1. Cont.

\begin{tabular}{|c|c|c|c|c|c|c|}
\hline $\begin{array}{l}\text { Study } \\
\text { (Country) }\end{array}$ & Aim of Study & PEA Type of Study & Population & $\mathbf{N}$ & $\begin{array}{l}\text { Outcome Measure } \\
\text { (Test Name or Description) }\end{array}$ & Results \\
\hline $\begin{array}{l}\text { Herrera et al. } \\
\text { (2018) (Spain) }\end{array}$ & $\begin{array}{l}\text { To assess the effect } \\
\text { of PEA on behavior } \\
\text { and neuronal } \\
\text { damage }\end{array}$ & $\begin{array}{l}\text { In vivo postnatal } \\
\text { exposure in animals }\end{array}$ & $\begin{array}{l}\text { 1. } 15 \text { PA+VHI; } 2.13 \\
\text { CTL (VHI); } 3.18 \\
\text { PA+PEA; } 4.17 \\
\text { CTL+PEA }\end{array}$ & 63 & $\begin{array}{l}\text { 1. Behavior (OFT, EPM); } 2 . \\
\text { neuronal damage (electron } \\
\text { microscopy, } \\
\text { immunohistochemistry (NeuN, } \\
\text { pNF-H/M, MAP-2, GFAP), } \\
\text { Western Blot (pNF H/M, MAP-2, } \\
\text { GFAP)) }\end{array}$ & $\begin{array}{l}\text { 1. OFT: time spent rearing, PA < CTL, PA < PA+PEA, } \\
\text { CTL vs. PA+PEA NS, CTL vs. CTL+PEA NS; time } \\
\text { spent grooming, PA > CTL, PA > PA+PEA, CTL vs. } \\
\text { PA+PEA NS, CTL vs. CTL+PEA NS; EPM: time spent } \\
\text { rearing, PA < CTL, PA < PA+PEA, CTL vs. PA+PEA } \\
\text { NS, CTL vs. CTL+PEA NS; time spent grooming, PA } \\
>\text { CTL, PA vs. PA+PEA NS, PA+PEA > CTL; time } \\
\text { spent HD, PA > CTL, PA > PA+PEA, CTL vs. } \\
\text { PA+PEA NS, CTL vs. CTL+PEA NS; } 2 \text {. pyknotic } \\
\text { nucleus in the hippocampal CA1 neurons: PA > } \\
\text { CTL, } \downarrow \text { in PA+PEA; NeuN abnormal neurons in the } \\
\text { hippocampal CA1 layer: PA > CTL, } \downarrow \text { in PA+PEA; } \\
\text { hippocampal pNF-H/M reactive area/ protein } \\
\text { expression: PA > CTL, } \downarrow \text { in PA+PEA (PA+PEA still > } \\
\text { CTL); hippocampal MAP-2reactive area/ protein } \\
\text { expression: PA < CTL, } \uparrow \text { in PA+PEA (PA+PEA still < } \\
\text { CTL); GFAP-positive cells and protein expression: NS }\end{array}$ \\
\hline
\end{tabular}


Table 1. Cont.

\begin{tabular}{|c|c|c|c|c|c|c|}
\hline $\begin{array}{l}\text { Study } \\
\text { (Country) }\end{array}$ & Aim of Study & PEA Type of Study & Population & $\mathbf{N}$ & $\begin{array}{l}\text { Outcome Measure } \\
\text { (Test Name or Description) }\end{array}$ & Results \\
\hline $\begin{array}{l}\text { Tomas-Roig } \\
\text { et al. (2018) } \\
\text { (Germany) }\end{array}$ & $\begin{array}{l}\text { To assess the effect } \\
\text { of repeated stress } \\
\text { and acute } \\
\text { cannabinoid } \\
\text { exposure on } \\
\text { behavior and } \\
\text { ECBs/AEs brain } \\
\text { levels }\end{array}$ & $\begin{array}{l}\text { Quantitative brain } \\
\text { assessment in animals }\end{array}$ & $\begin{array}{l}\text { 1. } 60 \text { STS; } 2.60 \text { CTL; } \\
\text { 3. VHI; } 4 \text {. WIN+VHI; } \\
\text { 5. Rim+VHI; } 6 . \\
\text { Rim+WIN; } \\
\text { 7.STS+VHI; } 8 . \\
\text { STS+WIN+VHI; } 9 . \\
\text { STS+Rim+VHI; } 10 . \\
\text { STS+Rim+WIN; } 11 . \\
\text { CTL+VHI; } 12 \text {. } \\
\text { CTL+WIN+VHI; } 13 . \\
\text { CTL+Rim+VHI; } 14 . \\
\text { CTL+Rim+WIN }\end{array}$ & 120 & $\begin{array}{l}\text { 1. Behavior (FOB, OFT); } 2 \text {. brain } \\
\text { ECBs / AEs and related gene } \\
\text { expression (LC-APCI-MS, } \\
\text { real-time PCR) }\end{array}$ & $\begin{array}{l}\text { 1. Effect of stress: AEA levels in dorsal CPu, STS < } \\
\text { CTL; Chrna6 gene expression, STS > CTL; } 2 \text {. Effect } \\
\text { of drugs exposure: OFT, distance travelled, } \\
\text { WIN+VHI < VHI, Rim+WIN < Rim+VHI; distance } \\
\text { travelled in center, Rim+VHI > WIN+VHI; rearing } \\
\text { activity, Rim+WIN > WIN+VHI; PEA and OEA } \\
\text { levels in dorsal Cpu, WIN+VHI > others; Fkpb5 } \\
\text { expression, Rim+WIN > others; 3. Effect of stress } \\
\text { under drug influence: OFT, distance travelled, } \\
\text { STS+VHI > CTL+VHI, STS/CTL+WIN+VHI < } \\
\text { STS/CTL+Rim+VHI, STS+WIN+VHI < STS+VHI, } \\
\text { CTL+Rim+VHI > CTL+VHI; 2-AG levels, } \\
\text { CTL+Rim+VHI > CTL+VHI/CTL+Rim+WIN; } \\
\text { Chrna6 gene expression: } \\
\text { CTL+WIN+VHI/CTL+Rim+VHI/CTL+Rim+WIN > } \\
\text { CTL+VHI, STS+Rim+VHI/STS+Rim+WIN > } \\
\text { STS+WIN+VHI/CTL groups; Slc6a4 gene } \\
\text { expression: Rim+VHI > others }\end{array}$ \\
\hline
\end{tabular}

$\begin{array}{llll}\begin{array}{l}\text { Udovin et al. } \\ \text { (2020) }\end{array} & \begin{array}{l}\text { To assess the effect } \\ \text { of PEA on neuronal }\end{array} & \begin{array}{l}\text { In vivo postnatal } \\ \text { exposure in animals }\end{array} & \begin{array}{l}\text { 1. 15 PA+VHI; } 2.13 \\ \text { CTL (VHI); } 3.18\end{array} \\ \text { (Argentina) } & \text { Pamage } & \text { CTL+PEA } 4.7\end{array}$

1. Neuronal damage (electron

microscopy,

immunohistochemistry

(pNF-H/M, MAP-2, GFAP)

Western Blot (pNF H/M, MAP-2,

GFAP,

antiglyceraldehyde-3-phosphate

dehydrogenase))

\section{Striatal $\mathrm{pNF}-\mathrm{H} / \mathrm{M}$ reactive area/protein} expression: PA $<\mathrm{CTL}, \uparrow$ in PA+PEA $(\mathrm{PA}+\mathrm{PEA}$ still $<$ CTL); striatal MAP-2 reactive area/protein

expression: PA $<$ CTL, $\uparrow$ in PA+PEA; GFAP-positive cells: PA < CTL, $\uparrow$ in PA+PEA (PA+PEA still < CTL; protein expression NS)

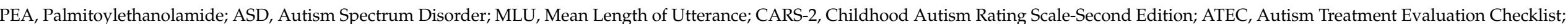

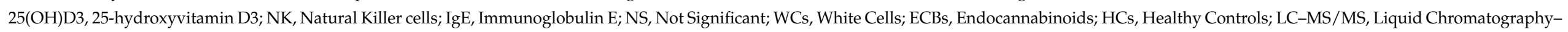

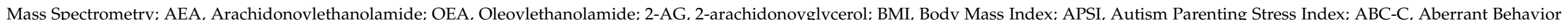

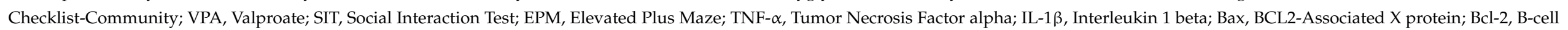

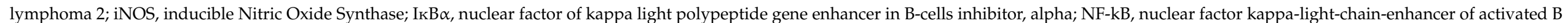

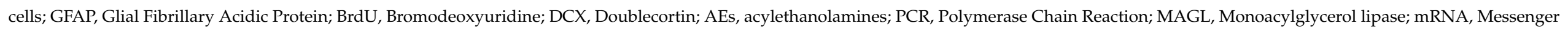

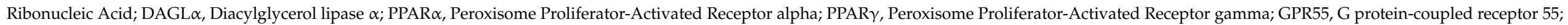

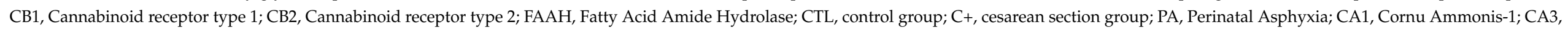




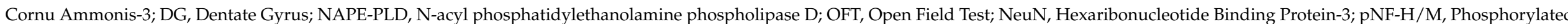

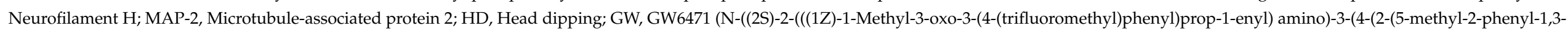

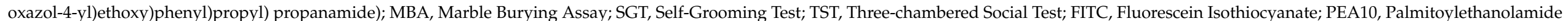

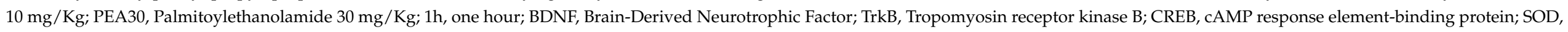

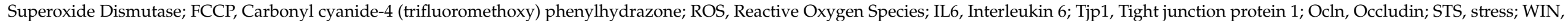

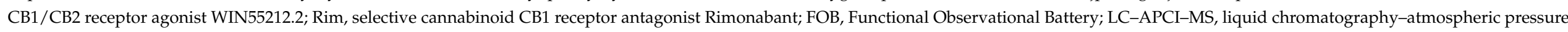

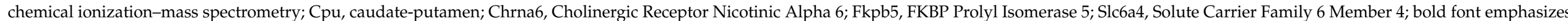
statistically significant results.

Table 2. Methodological quality of human studies investigating palmitoylethanolamide and its biobehavioral correlates in autism spectrum disorder.

\begin{tabular}{|c|c|c|c|c|c|c|c|c|c|c|c|c|}
\hline Study & Study Design & $\begin{array}{l}\text { Defined Study } \\
\text { Population }\end{array}$ & Age (Years) & Gender & PEA Measure & $\begin{array}{l}\text { Adequate PEA } \\
\text { Evaluation }\end{array}$ & Control & $\begin{array}{l}\text { Comparability of } \\
\text { Subjects }\end{array}$ & $\begin{array}{l}\text { Other } \\
\text { Comorbidity }\end{array}$ & $\begin{array}{l}\text { Excluded/Adjusted } \\
\text { for Confounding } \\
\text { Factors }\end{array}$ & $\begin{array}{l}\text { Statistical } \\
\text { Analyses }\end{array}$ & $\begin{array}{l}\text { Funding or } \\
\text { Sponsorship }\end{array}$ \\
\hline $\begin{array}{l}\text { Antonucci } \\
\text { et al. } \\
\text { (2015) } \\
\text { (Italy) }\end{array}$ & $\sqrt{ }$ Case report & $\begin{array}{l}\sqrt{ } \text { Clinical } \\
\text { diagnosis }\end{array}$ & $\begin{array}{l}\sqrt{ } 1.13 \text { years } \\
\text { old; } 2.15 \text { years } \\
\text { old }\end{array}$ & $\sqrt{ }$ Male & $\begin{array}{l}\sqrt{ } 1 \text {. Normast } \\
600 \mathrm{mg}, 1 / 2 \\
\text { tablet twice } \\
\text { daily for one } \\
\text { week, THEN } 1 \\
\text { tablet twice } \\
\text { daily (oral ad- } \\
\text { ministration); } \\
\text { 2. Normast } 600 \\
\text { mg, once daily } \\
\text { (oral adminis- } \\
\text { tration) }\end{array}$ & $\begin{array}{l}\sqrt{ } 1.1 \text { month } \\
\text { exposure; } 2.3 \\
\text { month } \\
\text { exposure }\end{array}$ & $x$ & NA & $\begin{array}{l}\sqrt{ } 1 \text {. Atopia; } 2 . \\
\text { Epilepsy }\end{array}$ & $x$ & NA & $\sqrt{ }$ \\
\hline $\begin{array}{l}\text { Aran et al. } \\
\text { (2019) } \\
\text { (Israel) }\end{array}$ & $\begin{array}{l}\sqrt{ } \text { Analytic, } \\
\text { observational }\end{array}$ & $\begin{array}{l}\sqrt{ } \text { ADOS-2, } \\
\text { DSM-5, } \\
\text { additional } \\
\text { assessments } \\
\text { (VABS-II, SCQ } \\
\text { lifetime form, } \\
\text { CARS2-ST, } \\
\text { HSQ-ASD, } \\
\text { CBCL- } \\
\text { validated } \\
\text { Hebrew } \\
\text { version, APSI, } \\
\text { SRS-2 Hebrew } \\
\text { version, CGI-S) }\end{array}$ & $\begin{array}{l}\sqrt{ } \text { Mean (SD) } \\
\text { [range]: } 1 . \mathrm{HC}: \\
11.8(4.3) \\
{[5.5-21] ; 2 .} \\
\text { ASD: 13.1 (4.1) } \\
\text { [6-21] }\end{array}$ & $\begin{array}{l}\sqrt{ } \text { Male }(\%): 1 . \\
\text { HC: } 79 \% ; 2 . \\
\text { ASD: } 79 \%\end{array}$ & $\begin{array}{l}\sqrt{ } \text { Serum } \\
\text { blood levels }\end{array}$ & $\begin{array}{l}\sqrt{ } \text { Single } \\
\text { assessment }\end{array}$ & $\sqrt{ }$ & $\begin{array}{l}\sqrt{ } \text { Matched for age, } \\
\text { gender and BMI }\end{array}$ & $\begin{array}{l}\sqrt{ } 1 \text {. HC, no } \\
\text { neuropsychi- } \\
\text { atric } \\
\text { comorbidity } \\
\text { other than } \\
\text { ADHD }\end{array}$ & $\begin{array}{l}\sqrt{ } 1 \text {. Results adjusted } \\
\text { for age, gender, BMI, } \\
\text { and ADHD; } 2 \text {. results } \\
\text { correlated with } \\
\text { anxiety, behavior, } \\
\text { epilepsy, and } \\
\text { perinatal } \\
\text { complication } \\
\text { comorbidity }\end{array}$ & $\begin{array}{l}\sqrt{ } \text { t-test, } \\
\text { Bonferroni } \\
\text { correction, } \\
\text { Pearson } \chi 2 \\
\text { test, Pearson } \\
\text { correlation, } \\
\text { multivariate } \\
\text { logistic } \\
\text { regression, } \\
\text { linear } \\
\text { regression }\end{array}$ & $\sqrt{ }$ \\
\hline $\begin{array}{l}\text { Bertolino } \\
\text { et al. } \\
\text { (2016) } \\
\text { (Italy) }\end{array}$ & $\sqrt{ }$ Case report & $\begin{array}{l}\sqrt{ } \text { Clinical } \\
\text { diagnosis }\end{array}$ & $\sqrt{ } 10$ years old & $\sqrt{ }$ Male & $\begin{array}{l}\sqrt{ } \text { co-ultraPEA- } \\
\text { Lut } 700 \mathrm{mg}+ \\
70 \mathrm{mg} \text { twice } \\
\text { daily (oral ad- } \\
\text { ministration) }\end{array}$ & $\begin{array}{l}\sqrt{ } 12 / 14 \\
\text { months }\end{array}$ & $x$ & NA & $\begin{array}{l}\sqrt{ } \text { Tetralogy of } \\
\text { Fallot }\end{array}$ & $x$ & NA & $\sqrt{ }$ \\
\hline
\end{tabular}


Table 2. Cont.

\begin{tabular}{|c|c|c|c|c|c|c|c|c|c|c|c|c|}
\hline Study & Study Design & $\begin{array}{l}\text { Defined Study } \\
\text { Population }\end{array}$ & Age (Years) & Gender & PEA Measure & $\begin{array}{l}\text { Adequate PEA } \\
\text { Evaluation }\end{array}$ & Control & $\begin{array}{l}\text { Comparability of } \\
\text { Subjects }\end{array}$ & $\begin{array}{l}\text { Other } \\
\text { Comorbidity }\end{array}$ & $\begin{array}{l}\text { Excluded/Adjusted } \\
\text { for Confounding } \\
\text { Factors }\end{array}$ & $\begin{array}{l}\text { Statistical } \\
\text { Analyses }\end{array}$ & $\begin{array}{l}\text { Funding or } \\
\text { Sponsorship }\end{array}$ \\
\hline $\begin{array}{l}\text { Khalaj } \\
\text { et al. } \\
\text { (2018) } \\
\text { (Iran) }\end{array}$ & $\begin{array}{l}\sqrt{ } \\
\text { Double-blind, } \\
\text { randomized } \\
\text { (parallel), } \\
\text { placebo } \\
\text { controlled }\end{array}$ & $\begin{array}{l}\sqrt{ } \text { DSM-5, } \\
\text { having } \\
\text { irritability } \\
\text { symptoms of } \\
\text { at least } \\
\text { moderate } \\
\text { severity } \\
\text { (scores } \geq 12 \text { on } \\
\text { the ABC-C } \\
\text { Irritability } \\
\text { subscale). }\end{array}$ & $\begin{array}{l}\sqrt{ } \text { Mean (SD) } \\
\text { [range]: } 1 . \\
\text { Risperidone + } \\
\text { PEA, 6.84 (2.1) } \\
\text { [4-12]; 2. } \\
\text { Risperidone + } \\
\text { placebo, 7.42 } \\
\text { (2.35) [4-12] }\end{array}$ & $\begin{array}{l}\sqrt{ } \text { Male, N (\%): } \\
\text { 1. Risperidone } \\
\text { + PEA, 22 } \\
\text { (70.97); } 2 . \\
\text { Risperidone + } \\
\text { placebo, 25 } \\
\text { (80.65) }\end{array}$ & $\begin{array}{l}\sqrt{ } 600 \mathrm{mg} \\
\text { twice daily } \\
\text { (oral adminis- } \\
\text { tration) }\end{array}$ & $\sqrt{ } 10$ weeks & $\sqrt{ }$ & $\begin{array}{l}\sqrt{ } \text { Matched for age, } \\
\text { sex, weight, ESRS } \\
\text { score, ABC-C } \\
\text { irritability, lethargy, } \\
\text { stereotypy, } \\
\text { hyperactivity, and } \\
\text { inappropriate } \\
\text { speech domains }\end{array}$ & $\begin{array}{l}\sqrt{ } \text { Exclusion } \\
\text { criterion }\end{array}$ & $\begin{array}{l}\sqrt{ } \text { Excluded if } 1 . \\
\text { symptoms not so } \\
\text { severe for treatment } \\
\text { with risperidone; } 2 . \\
\text { concomitant } \\
\text { psychiatric disorder; } 3 \text {. } \\
\text { preexisting medical } \\
\text { condition; } 4 \text {. severe } \\
\text { intellectual disability; } \\
\text { 5. alcohol/drug } \\
\text { abuse; } 6 \text {. dyskinesia; } \\
\text { 7. antipsychotic } \\
\text { medication or } \\
\text { behavior treatment } \\
\text { within the past } 6 \\
\text { months }\end{array}$ & $\begin{array}{l}\sqrt{ } \text { t-test with } \\
\text { Levene's test } \\
\text { for equality of } \\
\text { variance, } \\
\text { Freeman- } \\
\text { Halton } \\
\text { extension of } \\
\text { Fisher's exact } \\
\text { test, Cohen's d, } \\
\text { repeated } \\
\text { measures } \\
\text { ANOVA, ITT }\end{array}$ & $\sqrt{ }$ \\
\hline
\end{tabular}

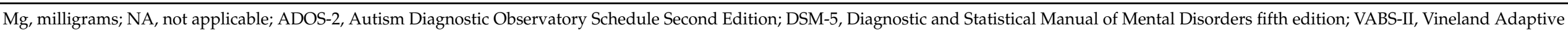

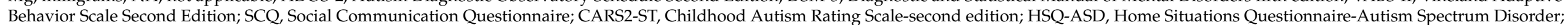

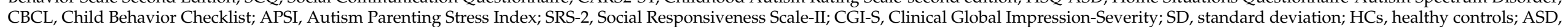

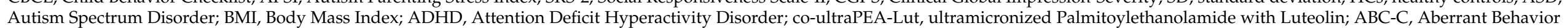

Checklist Community; PEA, Palmitoylethanolamide; ESRS, Extrapyramidal Symptom Rating Scale; ANOVA, Analysis of Variance; ITT, intention-to-treat.

Table 3. Methodological quality of animal studies investigating palmitoylethanolamide and its biobehavioral correlates in autism spectrum disorder

\begin{tabular}{|c|c|c|c|c|c|c|c|c|c|}
\hline Study & Study Design & $\begin{array}{l}\text { Defined Study } \\
\text { Population }\end{array}$ & Age & Gender & PEA Measure & $\begin{array}{l}\text { Adequate PEA } \\
\text { Evaluation }\end{array}$ & Control Group & Statistical Analyses & $\begin{array}{l}\text { Funding or } \\
\text { Sponsorship }\end{array}$ \\
\hline $\begin{array}{l}\text { Bertolino et al. } \\
\text { (2016) (Italy) }\end{array}$ & $\begin{array}{l}\sqrt{ } \text { Analytic, } \\
\text { observational, } \\
\text { interventional }\end{array}$ & $\begin{array}{l}\sqrt{ } \text { C57/BL6 mice } \\
\text { injected SC with } \\
\text { VPA }(400 \mathrm{mg} / \mathrm{kg}) \\
\text { on P14 }\end{array}$ & $\sqrt{ }$ P15-P120 & $\sqrt{ }$ Male & $\begin{array}{l}\sqrt{ } \\
\text { co-ultraPEA-LUT } \\
1 \mathrm{mg} / \mathrm{kg} \text { (oral } \\
\text { administration by } \\
\text { gavage) }\end{array}$ & $\begin{array}{l}\sqrt{ } 1.2 \text { weeks for } \\
\text { behavior, } \\
\text { immunochemistry } \\
\text { and Western Blot } \\
\text { studies; } 2.3 \\
\text { months for } \\
\text { neurogenesis } \\
\text { studies }\end{array}$ & $\begin{array}{l}\sqrt{ } \text { SHAM/VPA + } \\
\text { vehicle, } \\
\text { SHAM+PEA }\end{array}$ & $\begin{array}{l}\sqrt{ } \text { Behavior: one-way ANOVA, } \\
\text { Newman-Keuls multiple } \\
\text { comparison test; } \\
\text { Immunohistochemistry: ANOVA } \\
\text { and post hoc Tukey tests with } \\
\text { Bonferroni correction for multiple } \\
\text { comparisons; all other results: } \\
\text { ANOVA and Bonferroni post hoc } \\
\text { for multiple comparisons }\end{array}$ & $\sqrt{ }$ \\
\hline $\begin{array}{l}\text { Kerr et al. } \\
\text { (2013) } \\
\text { (Ireland) }\end{array}$ & $\begin{array}{l}\sqrt{ } \text { Analytic, } \\
\text { observational }\end{array}$ & $\begin{array}{l}\sqrt{ } \text { Litters of female } \\
\text { Sprague Dawley } \\
\text { rats SC injected } \\
\text { with VPA }(600 \\
\mathrm{mg} / \mathrm{kg}) \text { at } \mathrm{G} 12.5\end{array}$ & $\sqrt{ }$ P33-P40 & $\begin{array}{l}\sqrt{ } \text { Male and } \\
\text { female }\end{array}$ & $\begin{array}{l}\sqrt{ } \text { Brain tissue } \\
\text { levels }\end{array}$ & $\begin{array}{l}\sqrt{ } \text { Single } \\
\text { assessment }\end{array}$ & $\sqrt{ }$ Saline-treated & $\begin{array}{l}\sqrt{ } \text { Shapiro-Wilk test; Levene test; } \\
\text { unpaired t-test }\end{array}$ & $\sqrt{ }$ \\
\hline
\end{tabular}


Table 3. Cont

\begin{tabular}{|c|c|c|c|c|c|c|c|c|c|}
\hline Study & Study Design & $\begin{array}{l}\text { Defined Study } \\
\text { Population }\end{array}$ & Age & Gender & PEA Measure & $\begin{array}{l}\text { Adequate PEA } \\
\text { Evaluation }\end{array}$ & Control Group & Statistical Analyses & $\begin{array}{l}\text { Funding or } \\
\text { Sponsorship }\end{array}$ \\
\hline $\begin{array}{l}\text { Blanco et al. } \\
\text { (2015) (Spain) }\end{array}$ & $\begin{array}{l}\sqrt{ } \text { Analytic, } \\
\text { observational }\end{array}$ & $\begin{array}{l}\sqrt{ } \text { Rats exposed to } \\
\text { PA procedures }\end{array}$ & $\sqrt{ }$ P30 & $\sqrt{ }$ Male & $\begin{array}{l}\sqrt{ } \text { Brain tissue } \\
\text { levels of } \\
\text { PEA-related } \\
\text { enzymes and } \\
\text { receptors }\end{array}$ & $\begin{array}{l}\sqrt{ } \text { Single } \\
\text { assessment }\end{array}$ & $\sqrt{ } \mathrm{CTL}, \mathrm{C}+$ & $\begin{array}{l}\sqrt{ } \text { ANOVA, Tukey's post hoc tests } \\
\text { for multiple comparisons, } \\
\text { Bonferroni's correction, } \\
\text { Kuskal-Wallis test, Mann-Whitney } \\
\text { test }\end{array}$ & $\sqrt{ }$ \\
\hline $\begin{array}{l}\text { Herrera et al. } \\
\text { (2018) (Spain) }\end{array}$ & $\begin{array}{l}\sqrt{ } \text { Analytic, } \\
\text { observational, } \\
\text { interventional }\end{array}$ & $\begin{array}{l}\sqrt{ } \text { Rats exposed to } \\
\text { PA procedures }\end{array}$ & $\sqrt{ }$ P30 & $\sqrt{ }$ Male & $\begin{array}{l}\sqrt{ } 10 \mathrm{mg} / \mathrm{Kg}(\mathrm{SC} \\
\text { injection) }\end{array}$ & $\begin{array}{l}\sqrt{ } \text { Single } \\
\text { administration } \\
\text { (within the 1st h of } \\
\text { life) }\end{array}$ & $\begin{array}{l}\sqrt{ } \text { CTL/PA + } \\
\text { vehicle, CTL+PEA }\end{array}$ & $\begin{array}{l}\sqrt{ } \text { Shapiro-Wilk test; Levene test; } \\
\text { ANOVA; Student's t-test; } \\
\text { Bonferroni's correction }\end{array}$ & $\sqrt{ }$ \\
\hline $\begin{array}{l}\text { Cristiano et al. } \\
\text { (2018) (Italy) }\end{array}$ & $\begin{array}{l}\sqrt{ } \text { Analytic, } \\
\text { observational, } \\
\text { interventional }\end{array}$ & $\begin{array}{l}\sqrt{ } \text { BTBR T+tf/J } \\
\text { (BTBR) mice }\end{array}$ & $\begin{array}{l}\sqrt{ } 3-4 \\
\text { months }\end{array}$ & $\sqrt{ }$ Male & $\begin{array}{l}\sqrt{ } 10 \text { or } 30 \mathrm{mg} / \mathrm{Kg} \\
\text { (IP injection) }\end{array}$ & $\begin{array}{l}\sqrt{ } \text { Daily } \\
\text { administration (10 } \\
\text { days) }\end{array}$ & $\begin{array}{l}\sqrt{ } \\
\text { B6/BTBR/BTBR+GV } \\
+ \text { vehicle, } \\
\text { BTBR+GW/KO + } \\
\text { PEA }\end{array}$ & $\begin{array}{l}\text { V/KO } \\
\sqrt{ } \text { ANOVA, Bonferroni's correction }\end{array}$ & $\sqrt{ }$ \\
\hline $\begin{array}{l}\text { Tomas-Roig } \\
\text { et al. (2018) } \\
\text { (Germany) }\end{array}$ & $\begin{array}{l}\sqrt{ } \text { Analytic, } \\
\text { observational, } \\
\text { interventional }\end{array}$ & $\begin{array}{l}\sqrt{ } \mathrm{C} 57 \mathrm{BI} 6 / \mathrm{J} \text { mice } \\
\text { exposed to stress } \\
(1 \mathrm{~h} / \text { day per } 21 \\
\text { days })\end{array}$ & $\sqrt{ }$ 7-8 weeks & $\sqrt{ }$ Male & $\begin{array}{l}\sqrt{ } \text { Brain tissue } \\
\text { levels }\end{array}$ & $\begin{array}{l}\sqrt{ } \text { Single } \\
\text { assessment }\end{array}$ & $\begin{array}{l}\sqrt{ } \text { CTL (left } \\
\text { undisturbed) }\end{array}$ & $\begin{array}{l}\sqrt{ } \text { ANOVA, Brown-Forsythe test, } \\
\text { Bonferroni's correction, Tamhane } \\
\text { post hoc test, Student's t-test }\end{array}$ & $\sqrt{ }$ \\
\hline
\end{tabular}

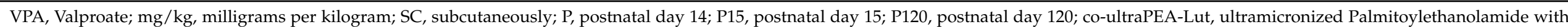

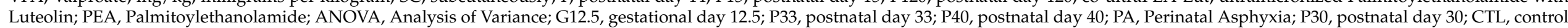

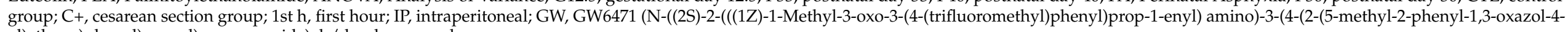
yl)ethoxy)phenyl)propyl) propanamide); h/day, hour per day 


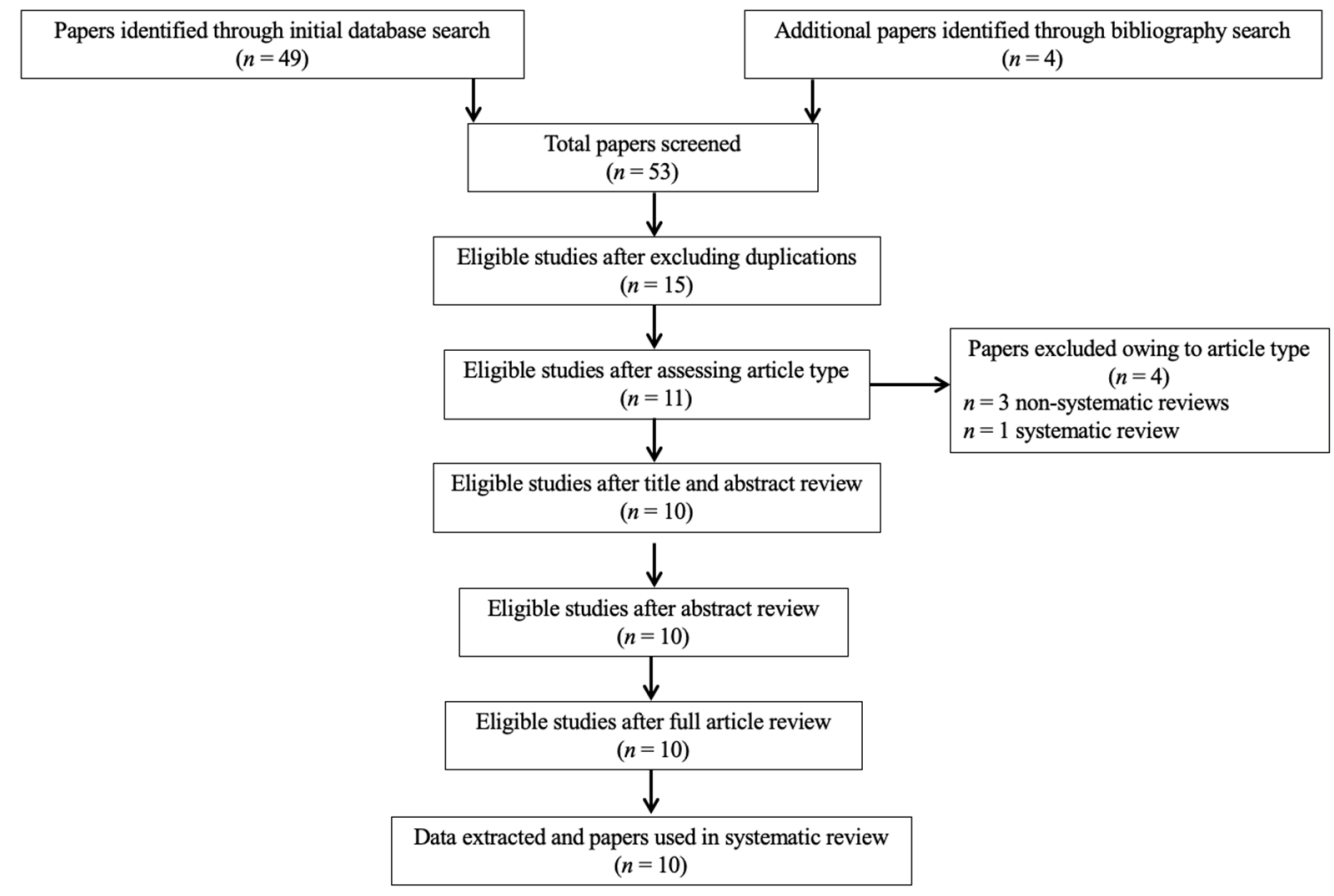

Figure 1. PRISMA flowchart of search strategy for systematic review.

\subsection{In Vivo PEA Treatment Exposure in Children and Adolescents with Autism Spectrum Disorder}

Three studies have addressed this area in humans, including two case reports of two adolescents [25] and one child [26] with ASD as well as a double-blind, randomized (parallel), placebo-controlled trial performed among 62 risperidone-treated ASD children [27]. These studies have assessed the effects of PEA treatment on language [25,27], behavior [25-27] and additional parameters. The two case reports indicated that PEA supplementation improves expressive language [25] and reduces overall autism severity [25,26], including motor stereotypic behaviors [26], appearing to be beneficial also in modulating immune chemistry [25] and controlling enuresis [26]. The trial of PEA add-on to risperidone reported significant improvements in the domains of irritability and hyperactivity among ASD children receiving the combination treatment of risperidone and PEA as compared to those receiving only risperidone, with weak effects of PEA add-on also in reducing stereotypic behavior and inappropriate speech [27].

\subsection{PEA Levels in Children and Adolescents with Autism Spectrum Disorder as Compared to Healthy Controls}

This systematic review identified a single study specifically investigating whether ASD children and adolescents differ from their healthy peers in terms of circulating blood levels of PEA and other endocannabinoids (ECBs)/acylethanolamines (AEs). This study found that ASD children and adolescents have lower serum levels of PEA and other ECBs/AEs as compared to healthy controls. Additionally, PEA levels did not appear to change as a function of any sociodemographic and clinical characteristics [28].

\subsection{In Vivo PEA Postnatal Exposure in Animal Models of Autism and Perinatal Brain Disorders}

All these studies investigated the effect of PEA exposure, using similar but not overlapping methodologies in terms of animal type (mice [26,29], rat [30,31]), mode of administration (oral [26], subcutaneous [30,31], intraperitoneal [29]), period of exposure (postnatal day P15 to P120), dosage of PEA (1 to $30 \mathrm{mg} / \mathrm{kg}$ ), and model of pathology (valproic acid 
(VPA) exposure [26], perinatal asphyxia (PA) [30,31], BTBR T+tf/J strain (BTBR) [29]). The first of these studies indicated that PEA administration improves social and nonsocial behaviors, reduces the expression of proinflammatory markers, modulates apoptosis in the hippocampus and cerebellum, and increases hippocampal neurogenesis and neuroplasticity in the VPA-induced rodent model of autism [26]. Another study found that PEA administration reverts the altered social and nonsocial behavioral phenotype in the inbred BTBR strain used as mice model of autism, possibly by reducing the expression of pro-inflammatory markers, activating the peroxisome proliferator-activated receptor- $\alpha$ (PPAR- $\alpha$ ), and restoring hippocampal brain derived neurotrophic factor (BDNF) signaling, mitochondrial function, and microbiota composition [29]. Subsequent studies investigated the potential neuroprotective role of PEA in the PA rat model at P30 [30,31], since this postnatal age would correspond to the age of onset of neurodevelopmental conditions in humans. PEA treatment within the first hour of life attenuated the PA-induced alterations at P30 such as the altered prototypical behaviors [30], as well as the biochemical and morphological signs of altered neuronal cytoskeleton [30,31] and degeneration [30] in the hippocampus [30] and striatum [31].

3.5. Pea and Related Enzymes and Receptors Quantitative Brain Assessment in Animal Models of Autism, Perinatal Brain Disorders, and Stress Following Exposure to Exogenous Cannabinoids

In total, three studies did not evaluate the direct effect of PEA exposure while analyzing PEA levels and indirect measures of PEA signaling in the brain of animal models of autism and related disturbances [32-34]. A study found that alterations in various components of the endocannabinoid system could explain the autistic-like behavior observed among rats prenatally exposed to VPA, including increased hippocampal levels of PEA and other ECBs/AEs in response to social exposure, altered expression and activity of the enzymes responsible for the synthesis and catalysis of the 2-arachidonoylglycerol (2-AG) and reduced expression of PPAR- $\alpha$ and GPR55 mRNA across different brain areas [32]. Similarly, another study found that the behavioral alterations observed in the PA rat model could be due to a dysregulation of the enzymes responsible for the synthesis and catalysis of ECBs/AEs and a reduction in the expression of PPAR- $\alpha$ in the hippocampus [33]. Finally, a study found that both stress and exogenous manipulation of the endocannabinoid system affect ECBs/AEs levels and expression of candidate genes for neuropsychiatric disorders such as the cholinergic receptor nicotinic alpha 6 (Chrna6) and serotonin transporter neurotransmitter type 4 (Slc6a4) genes, with implications for the manifestation of aberrant behaviors [34].

\section{Discussion}

This is the first systematic review of all studies exploring the biobehavioral correlates of palmitoylethanolamide (PEA) in autism spectrum disorder (ASD) in humans and animals. Previous reviews have mainly addressed the potential role of neuroinflammation and altered glutamate signaling in ASD etiopathogenesis, indicating that, in subjects with genetic predispositions, atypical neurodevelopment may arise from a complex interplay between (neuro)inflammatory processes, mitochondrial dysfunction, oxidative stress and altered expression of glutamate signaling [35]. Interestingly, research evidence converges on the crucial role of exogenous cannabinoids and endocannabinoids in modulating such neurobiological systems, including neuroinflammation [12] and glutamate neurotransmission [13]. Overall, this review demonstrates that PEA may be involved in ASD, as indicated by interventional studies of the positive biobehavioral effects of PEA supplementation in both humans and animals as well as observational studies reporting aberrancies in the PEA signaling pathway at different levels.

PEA supplementation in humans as both monotherapy $[25,26]$ and add-on therapy to antipsychotic medication [27] has been shown to reduce overall autism severity [25,26] by improving both expressive language (what the child says) [25] and inappropriate speech (how the child says it) [27], as well as modulating atypical behavior [25-27] and immune response [25]. Similarly, PEA supplementation in different animal models of autism and 
related conditions has been suggested to be effective in improving social and nonsocial behaviors $[26,29,30]$ as well as in modulating a number of neurobiological processes including neuroinflammation [26], neurotrophy [29], apoptosis [26], neurogenesis [26], neuroplasticity [26], neurodegeneration [30,31], mitochondrial function [29], and microbiota activity [29]. Importantly, PEA administration resulted in an activation of the peroxisome proliferator activated receptor- $\alpha$ (PPAR- $\alpha$ ) [29], whose downregulation may decrease antioxidative and anti-inflammatory processes, also altering energy homeostasis, mitochondrial fatty acid metabolism, and regulation of genes coding proteins that are involved in glutamate homeostasis and cholinergic/dopaminergic signaling in the brain, with implications for neurodevelopmental conditions [36]. The hippocampus is one of the brain areas where such modulatory effects of PEA are mostly reported. Further studies are needed to investigate the role of the PEA-induced modulation of the hippocampal system in improving the brain spatiotemporal framework within which sensory, emotional, and cognitive aspects of an experience are processed in ASD, with implications for comprehension and language production [37]. This is of paramount importance, as structural, functional, and neurochemical alterations in the hippocampus have been suggested as candidate biomarkers for the diagnosis of ASD in childhood [38].

Another line of research identified lower serum levels of PEA and other endocannabinoids (ECBs)/acylethanolamines (AEs) in humans suffering from ASD, independently of sociodemographic and clinical characteristics [28]. Similarly, studies performed in animal models of autism and related conditions suggested an altered response of the PEA signaling in the face of social exposure [32], altered expression and activity of the enzymes responsible for the synthesis and catalysis of ECBs/AEs [32,33], and downregulation of PPAR- $\alpha[32,33]$ and the cannabinoid receptor target GPR55 mRNA expression in the brain [32]. Interestingly, animals exposed to stress and administered with exogenous cannabinoids showed variations in their ECB/AE levels and expression of genes implied in neuropsychiatric disorders [34].

The findings of this systematic review have to be seen in light of some limitations. Research in the field is still too limited, especially in humans (2 of the 4 human studies are case reports, leaving a single experimental study conducted in a youth population with ASD), and further studies are needed to fully address the relevance of PEA for the different clinical phenotypes of ASD and whether the potentially beneficial effects of PEA are mediated by a protective role of the compound against altered neuroinflammatory responses and glutamate toxicity, which have been suggested to be involved in the pathophysiology of ASD [7-10]. Additionally, whether the PEA concentration profile can be used as a biomarker for ASD remains to be tested, and future longitudinal studies will have to investigate its clinical utility in monitoring response to treatment. Moreover, as the available evidence does not allow excluding a placebo effect [39], further clinical trials in larger samples are needed to fully explore the efficacy and tolerability of PEA supplementation in ASD. Finally, even though the systematic review followed the PRISMA statement, no review protocol was registered.

\section{Conclusions}

This review revealed a paucity of observational and experimental investigations of PEA and its pathway in ASD. However, the 10 studies discussed here seem to converge in reporting alterations of the PEA signaling, implications for ASD-related biobehavioral manifestations, and benefits from PEA supplementation. In particular, PEA may be useful in improving language difficulties and stereotypic behavior as well as in controlling hyperactivity and irritability which co-occur frequently in ASD. Notably, no serious adverse effects were observed with the administration of the compound in all human studies reviewed here, making PEA supplementation a potentially valid and reasonably safe therapeutic intervention in ASD. 
Author Contributions: Conceptualization, M.C., R.B., R.C. and L.Z.; methodology, M.C., R.B., R.C. and L.Z.; validation, M.C., R.B., R.C. and L.Z.; investigation, M.C. and R.B.; resources, M.C., R.B., R.C. and L.Z.; data curation, M.C., R.B., R.C. and L.Z.; writing-original draft preparation, M.C., R.B., and R.C.; writing-review and editing, M.C., R.B., R.C. and L.Z.; visualization, M.C., R.B., R.C. and L.Z.; supervision, M.C.; project administration, M.C. All authors have read and agreed to the published version of the manuscript.

Funding: This research received no external funding.

Institutional Review Board Statement: Not applicable.

Informed Consent Statement: Not applicable.

Data Availability Statement: Not applicable.

Acknowledgments: The authors would like to acknowledge infrastructure from the Integrated University Hospital of Verona and the University of Verona.

Conflicts of Interest: M.C. has been a consultant/advisor to GW Pharma Limited outside of this work. All the other authors declare no conflict of interest.

\section{References}

1. Saghazadeh, A.; Ahangari, N.; Hendi, K.; Saleh, F.; Rezaei, N. Status of essential elements in autism spectrum disorder: Systematic review and meta-analysis. Rev. Neurosci. 2017, 28, 783-809. [CrossRef]

2. First, M.B.; Williams, J.B.W.; Karg, R.S.; Spitzer, R.L. Structured Clinical Interview for DSM-5 Disorders, Clinician Version (SCID-5-CV); American Psychiatric Association: Arlington, VA, USA, 2015.

3. Zeng, K.; Kang, J.; Ouyang, G.; Li, J.; Han, J.; Wang, Y.; Sokhadze, E.M.; Casanova, M.F.; Li, X. Disrupted Brain Network in Children with Autism Spectrum Disorder. Sci. Rep. 2017, 7, 1-12. [CrossRef]

4. Kern, J.K.; Geier, D.A.; King, P.G.; Sykes, L.K.; Mehta, J.A.; Geier, M.R. Shared Brain Connectivity Issues, Symptoms, and Comorbidities in Autism Spectrum Disorder, Attention Deficit/Hyperactivity Disorder, and Tourette Syndrome. Brain Connect. 2015, 5, 321-335. [CrossRef]

5. Lukito, S.; Norman, L.; Carlisi, C.; Radua, J.; Hart, H.; Simonoff, E.; Rubia, K. Comparative meta-analyses of brain structural and functional abnormalities during cognitive control in attention-deficit/hyperactivity disorder and autism spectrum disorder. Psychol. Med. 2020, 50, 894-919. [CrossRef]

6. Dewey, D. What Is Comorbidity and Why Does It Matter in Neurodevelopmental Disorders? Curr. Dev. Disord. Rep. 2018, 5, 235-242. [CrossRef]

7. Masi, A.; Quintana, D.S.; Glozier, N.; Lloyd, A.R.; Hickie, I.B.; Guastella, A.J. Cytokine aberrations in autism spectrum disorder: A systematic review and meta-analysis. Mol. Psychiatry 2014, 20, 440-446. [CrossRef] [PubMed]

8. Purcell, A.E.; Jeon, O.H.; Zimmerman, A.W.; Blue, M.E.; Pevsner, J. Postmortem brain abnormalities of the glutamate neurotransmitter system in autism. Neurology 2001, 57, 1618-1628. [CrossRef] [PubMed]

9. Shimmura, C.; Suda, S.; Tsuchiya, K.J.; Hashimoto, K.; Ohno, K.; Matsuzaki, H.; Iwata, K.; Matsumoto, K.; Wakuda, T.; Kameno, Y.; et al. Alteration of Plasma Glutamate and Glutamine Levels in Children with High-Functioning Autism. PLoS ONE 2011, 6, e25340. [CrossRef]

10. Shinohe, A.; Hashimoto, K.; Nakamura, K.; Tsujii, M.; Iwata, Y.; Tsuchiya, K.J.; Sekine, Y.; Suda, S.; Suzuki, K.; Sugihara, G.-I.; et al. Increased serum levels of glutamate in adult patients with autism. Prog. Neuro Psychopharmacol. Biol. Psychiatry 2006, 30, 1472-1477. [CrossRef] [PubMed]

11. Blaylock, R.L.; Strunecka, A. Immune-glutamatergic dysfunction as a central mechanism of the autism spectrum disorders. Curr. Med. Chem. 2009, 16, 157-170. [CrossRef] [PubMed]

12. Walter, L.; Stella, N. Cannabinoids and neuroinflammation. Br. J. Pharmacol. 2004, 141, 775-785. [CrossRef]

13. Colizzi, M.; McGuire, P.; Pertwee, R.G.; Bhattacharyya, S. Effect of cannabis on glutamate signalling in the brain: A systematic review of human and animal evidence. Neurosci. Biobehav. Rev. 2016, 64, 359-381. [CrossRef]

14. Colizzi, M.; Ruggeri, M.; Bhattacharyya, S. Unraveling the Intoxicating and Therapeutic Effects of Cannabis Ingredients on Psychosis and Cognition. Front. Psychol. 2020, 11, 833. [CrossRef]

15. Freitas, H.R.; Isaac, A.R.; Malcher-Lopes, R.; Diaz, B.L.; Trevenzoli, I.H.; Reis, R.A.D.M. Polyunsaturated fatty acids and endocannabinoids in health and disease. Nutr. Neurosci. 2017, 21, 695-714. [CrossRef]

16. Aran, A.; Harel, M.; Cassuto, H.; Polyansky, L.; Schnapp, A.; Wattad, N.; Shmueli, D.; Golan, D.; Castellanos, F.X. Cannabinoid treatment for autism: A proof-of-concept randomized trial. Mol. Autism 2021, 12, 1-11. [CrossRef]

17. Tsuboi, K.; Uyama, T.; Okamoto, Y.; Ueda, N. Endocannabinoids and related N-acylethanolamines: Biological activities and metabolism. Inflamm. Regen. 2018, 38, 1-10. [CrossRef]

18. Rankin, L.; Fowler, C.J. The basal pharmacology of palmitoylethanolamide. Int. J. Mol. Sci. 2020, 21, 7942. [CrossRef] [PubMed] 
19. Solorzano, C.; Zhu, C.; Battista, N.; Astarita, G.; Lodola, A.; Rivara, S.; Mor, M.; Russo, R.; Maccarrone, M.; Antonietti, F.; et al. Selective $\mathrm{N}$-acylethanolamine-hydrolyzing acid amidase inhibition reveals a key role for endogenous palmitoylethanolamide in inflammation. Proc. Natl. Acad. Sci. USA 2009, 106, 20966-20971. [CrossRef] [PubMed]

20. Verme, J.L.; Fu, J.; Astarita, G.; La Rana, G.; Russo, R.; Calignano, A.; Piomelli, D. The nuclear receptor peroxisome proliferatoractivated receptor- $\alpha$ mediates the anti-inflammatory actions of palmitoylethanolamide. Mol. Pharmacol. 2005, 67, 15-19. [CrossRef] [PubMed]

21. Yu, H.-L.; Deng, X.-Q.; Li, Y.-J.; Quan, Z.-S.; Sun, X.-Y.; Li, Y.-C. Short communication-N-palmitoylethanolamide, an endocannabinoid, exhibits antidepressant effects in the forced swim test and the tail suspension test in mice. Pharmacol. Rep. 2011, 63, 834-839. [CrossRef]

22. Lambert, D.M.; Vandevoorde, S.; Diependaele, G.; Govaerts, S.J.; Robert, A.R. Anticonvulsant activity of N-palmitoylethanolamide, a putative endocannabinoid, in mice. Epilepsia 2002, 42, 321-327. [CrossRef]

23. Jaggar, S.I.; Hasnie, F.S.; Sellaturay, S.; Rice, A.S. The anti-hyperalgesic actions of the cannabinoid anandamide and the putative CB2 receptor agonist palmitoylethanolamide in visceral and somatic inflammatory pain. Pain 1998, 76, 189-199. [CrossRef]

24. West, S.; King, V.; Carey, T.S.; Lohr, K.N.; McKoy, N.; Sutton, S.F.; Lux, L. Systems to rate the strength of scientific evidence. Évid. Rep. Assess. 2002, 47, 1-11.

25. Antonucci, N.; Cirillo, A.; Siniscalco, D. Beneficial Effects of Palmitoylethanolamide on Expressive Language, Cognition, and Behaviors in Autism: A Report of Two Cases. Case Rep. Psychiatry 2015, 2015, 1-6. [CrossRef] [PubMed]

26. Bertolino, B.; Crupi, R.; Impellizzeri, D.; Bruschetta, G.; Cordaro, M.; Siracusa, R.; Esposito, E.; Cuzzocrea, S. Beneficial Effects of Co-Ultramicronized Palmitoylethanolamide/Luteolin in a Mouse Model of Autism and in a Case Report of Autism. CNS Neurosci. Ther. 2016, 23, 87-98. [CrossRef] [PubMed]

27. Khalaj, M.; Saghazadeh, A.; Shirazi, E.; Shalbafan, M.-R.; Alavi, K.; Shooshtari, M.H.; Laksari, F.Y.; Hosseini, M.; Mohammadi, M.-R.; Akhondzadeh, S. Palmitoylethanolamide as adjunctive therapy for autism: Efficacy and safety results from a randomized controlled trial. J. Psychiatr. Res. 2018, 103, 104-111. [CrossRef]

28. Aran, A.; Eylon, M.; Harel, M.; Polianski, L.; Nemirovski, A.; Tepper, S.; Schnapp, A.; Cassuto, H.; Wattad, N.; Tam, J. Lower circulating endocannabinoid levels in children with autism spectrum disorder. Mol. Autism 2019, 10, 1-11. [CrossRef]

29. Cristiano, C.; Pirozzi, C.; Coretti, L.; Cavaliere, G.; Lama, A.; Russo, R.; Lembo, F.; Mollica, M.P.; Meli, R.; Calignano, A.; et al. Palmitoylethanolamide counteracts autistic-like behaviours in BTBR T+tf/J mice: Contribution of central and peripheral mechanisms. Brain Behav. Immun. 2018, 74, 166-175. [CrossRef]

30. Herrera, M.I.; Udovin, L.D.; Toro-Urrego, N.; Kusnier, C.F.; Luaces, J.P.; Capani, F. Palmitoylethanolamide Ameliorates Hippocampal Damage and Behavioral Dysfunction After Perinatal Asphyxia in the Immature Rat Brain. Front. Neurosci. 2018, 12, 145. [CrossRef]

31. Udovin, L.D.; Kobiec, T.; Herrera, M.I.; Toro-Urrego, N.; Kusnier, C.F.; Kölliker-Frers, R.A.; Ramos-Hryb, A.B.; Luaces, J.P.; Otero-Losada, M.; Capani, F. Partial Reversal of Striatal Damage by Palmitoylethanolamide Administration Following Perinatal Asphyxia. Front. Neurosci. 2020, 13, 1345. [CrossRef]

32. Kerr, D.; Downey, L.; Conboy, M.; Finn, D.; Roche, M. Alterations in the endocannabinoid system in the rat valproic acid model of autism. Behav. Brain Res. 2013, 249, 124-132. [CrossRef]

33. Blanco, E.; Galeano, P.; Holubiec, M.I.; Romero, J.I.; Logica, T.; Rivera, P.; Pavón, F.J.; Suarez, J.; Capani, F.; De Fonseca, F.R. Perinatal asphyxia results in altered expression of the hippocampal acylethanolamide/endocannabinoid signaling system associated to memory impairments in postweaned rats. Front. Neuroanat. 2015, 9, 141. [CrossRef]

34. Tomas-Roig, J.; Piscitelli, F.; Gil, V.; Quintana, E.; Ramió-Torrentà, L.L.; Del Río, J.A.; Moore, T.P.; Agbemenyah, H.; Salinas, G.; Pommerenke, C.; et al. Effects of repeated long-term psychosocial stress and acute cannabinoid exposure on mouse corticostriatal circuitries: Implications for neuropsychiatric disorders. CNS Neurosci. Ther. 2018, 24, 528-538. [CrossRef] [PubMed]

35. Savino, R.; Carotenuto, M.; Polito, A.N.; Di Noia, S.; Albenzio, M.; Scarinci, A.; Ambrosi, A.; Sessa, F.; Tartaglia, N.; Messina, G. Analyzing the Potential Biological Determinants of Autism Spectrum Disorder: From Neuroinflammation to the Kynurenine Pathway. Brain Sci. 2020, 10, 631. [CrossRef] [PubMed]

36. Wójtowicz, S.; Strosznajder, J.B.; Jeżyna, M. The Novel Role of PPAR Alpha in the Brain: Promising Target in Therapy of Alzheimer's Disease and Other Neurodegenerative Disorders. Neurochem. Res. 2020, 45, 972-988. [CrossRef] [PubMed]

37. Hay, I.; Hynes, K.L.; Burgess, J.R. Mild-to-Moderate Gestational Iodine Deficiency Processing Disorder. Nutrients 2019, 11 , 1974. [CrossRef] [PubMed]

38. Li, D.; Karnath, H.-O.; Xu, X. Candidate Biomarkers in Children with Autism Spectrum Disorder: A Review of MRI Studies. Neurosci. Bull. 2017, 33, 219-237. [CrossRef]

39. Siafis, S.; Çıray, O.; Schneider-Thoma, J.; Bighelli, I.; Krause, M.; Rodolico, A.; Ceraso, A.; Deste, G.; Huhn, M.; Fraguas, D.; et al. Placebo response in pharmacological and dietary supplement trials of autism spectrum disorder (ASD): Systematic review and meta-regression analysis. Mol. Autism 2020, 11, 1-19. [CrossRef] 